\title{
Collision Tumors of the Ovary: Dermoid Cysts with Borderline Epithelial Ovarian Tumor with Metachronous Invasive Epithelial Carcinoma of Ovary: A Case Series
}

\author{
Ab Wahid Mir, M.S. 'Syed Besina Yasin, M.D. ${ }^{2}$ Syed Arshad Mustafa, M.D. ${ }^{3}$ Irfan Robbani, M.D ${ }^{4}$ \\ ' Associate Professor, Department of Surgical Oncology, SKIMS \\ ${ }^{2}$ Professor, Department of Pathology, SKIMS \\ ${ }^{3}$ Associate Professor, Department of Radiation Oncology, GMC, Srinagar, Kashmir, India. \\ ${ }^{4}$ Professor, Department of Radio diagnosis Imaging, SKIMS
}

\section{A B S T R A C T}

A Collision tumor is defined as the coexistence of two or more histologically distinct tumors adjacent to each other in the same organ without any histological intermixing. Collision tumors have been described to occur in many body organs, yet its occurrence in ovary is extremely rare. In our series of two patients, one had a dermoid cyst was co-existing with borderline epithelial ovarian tumor, which is the rarest of rare variety of collision tumors reported in ovary. The other patient had a dermoid cyst with recurrent invasive epithelial ovarian malignancy. JMS 2018; 21 (1):37-39

Keywords: Collision tumor, Ovary, Dermoid Cyst, Epithelial Carcinoma.

\section{INTRODUCTION}

Collision tumor is the occurrence of histologically two or more distinct tumors in the same organ or tissue, without evidence of any histological intermixture. Such collision tumors have been reported in various body organs including esophagus, stomach, thyroid, and many more; yet the occurrence of a collision tumor in ovary is an extremely rare phenomenon $^{[1,2]}$.

Ovarian collision tumors as described are invariably composed of teratoma and cystadenoma or cystadenocarcinoma $^{[3]}$. On scrutiny of literature, we found that the collision tumors of the ovary have been classically described with the following histopathological features: cystadenocarcinoma and granulosa cell tumor ${ }^{[4]}$, teratoma with granulosa cell tumor ${ }^{[5]}$, and serous adenocarcinoma with steroid cell tumor ${ }^{[6]}$.

In the study conducted at the Seoul National University College of Medicine only one case of borderline tumor in combination with teratoma as collision tumor has reported $^{[7]}$. In our institution, we have encountered two interesting cases of collision tumors of ovary occurring as

\section{Correspondence}

Dr. Irfan Robbani, M.D.

Professor, Department of Radio diagnosis, SKIMS

E-mail: irfanrobbani@yahoo.co.in combination of teratoma with borderline tumors. This is extremely rare, and we herein present the twin cases.

\section{Case Report:}

Case 1:

A 55-year-old post-menopausal hypertensive female presented with complaints of fullness of abdomen since one month. There was past history of cholecystectomy. On examination, the patient was found to have a large palpable mass arising from the pelvis and nearly occupying whole of the abdomen and extending superiorly up to the epigastric region. USG showed a large cystic lesion measuring $25 \times 23$ $\mathrm{x} 20 \mathrm{~cm}$ occupying the pelvis and extending into the abdomen; the lesion showed multiple linear echoes and solid intralesional components. The patient next underwent CECT examination which revealed a large complex cystic lesion with another lipid laden subjacent lesion showing features consistent with a possible dermoid cyst. The patients CA125 was $63.80 \mathrm{U} / \mathrm{mL}$ and CEA stood at 30.39 $\mathrm{ng} / \mathrm{mL}$. Endoscopic and colonoscopic studies were essentially normal. A staging laparotomy comprising abdominal hysterectomy, bilateral salpingo-oophorectomy, omentectomy, appendectomy and multiple peritoneal biopsies was performed (Fig.1). Intra-operative findings revealed a large cystic lesion arising from the right ovary with intact capsule. Rest of the laparotomy was grossly normal. Histopathological examination showed borderline 
mucinous tumor of the ovary with adjacent dermoid cyst (Fig.2). After one month her CA 125 levels decreased to $11.01 \mathrm{U} / \mathrm{mL}$. In view of borderline pathology, it was deemed unnecessary to subject her to adjuvant chemotherapy; the patient is doing fine and is under our regular follow up since last two years.

\section{Case 2:}

A 25-year-old female had presented with pain of lower abdomen of about one-month duration along the lower right quadrant of the abdomen. On examination, a mass was palpable in the pelvis extending up to the umbilicus. Past history revealed that she had undergone a surgical procedure for similar complaints about 4 years back.

The patient's records revealed that she had been previously subjected to ultrasonography examination (USG), which had revealed a large predominantly cystic lesion with some intra-lesional strands; the lesion measured $15 \times 12 \times 14 \mathrm{~cm}$ and was not seen separately from the ipsilateral ovary. Routine laboratory investigations conducted that time were largely normal. The patient had also undergone contrast enhanced Computed Tomography (CECT) which had demonstrated presence of complex cystic lesion arising in the right lower abdomen, crossing the midline and mildly pushing the urinary bladder towards the contralateral side; this was thought to be arising from the right ovary. The $\mathrm{Ca}$ 125 had been recorded as $141.50 \mathrm{U} / \mathrm{mL}$; the serum AlphaFetoprotein and Beta Human Chorionic Gonadotropin (HCG) levels were also normal at $1.61 \mathrm{ng} / \mathrm{mL}$ and 1.2 $\mathrm{mIU} / \mathrm{mL}$, respectively. The patient had undergone laparotomy and intra-operative findings had revealed a $15 \times 15 \mathrm{~cm}$ cystic lesion, with another adjacent cystic lesion measuring $6 \times 8 \mathrm{~cm}$, arising from right ovary; there was no identifiable ovarian parenchyma. The patient had undergone right salpingo-oophorectomy, without proper staging by the surgeon. Histopathological examination had revealed a large cyst showing features of borderline papillary serous carcinoma with another separate adjacent cyst showing presence of cheesy material along with hair, suggestive of a dermoid arising from the ovary. Patient had accordingly been put on observation.

The patient stopped her follow-up after one year; three years later she was now presenting to the Surgical Oncology OPD with complaints mentioned here in above. After examination she underwent USG which revealed a large pelvic cyst about $10 \mathrm{~cm}$ in diameter. The cyst contained some solid elements, as well. CECT revealed a large complex predominantly cystic pelvic mass eccentrically located towards the right side and containing a few enhancing solid nodules; the mass measured $14 \times 13 \times 10 \mathrm{~cm}$ in size. Tumor markers (CA 125, CEA, AFP, Beta HCG) were normal. Patient was planned for surgery. The patient was planned for exploratory laparotomy which revealed a recurrent predominantly cystic lesion in in the right ovarian fossa with possible infiltration into the sigmoid colon and engulfing the subjacent appendix. The omentum was adherent to pelvis. Contralateral ovary also showed presence of $5 \times 4 \mathrm{~cm}$ cystic lesion. We performed total abdominal hysterectomy with excision of the right ovarian mass along with left salpingo-oophorectomy along with sigmoid colectomy and appendectomy en-mass. In addition, infra-colic omentectomy, bilateral pelvic lymph node dissection and para-aortic lymph node dissection was also done. Histopathology examination showed features of invasive papillary serous cystadenocarcinoma involving both ovaries with two pelvic lymph node positive for malignancy. The patient was staged as Ca ovary stage IIIC and was referred for adjuvant chemotherapy. Till filing of this report, the patient was doing well.

\section{DISCUSSION}

Collision tumors have been reported in several organs, however, collision tumors of the ovary are extremely rare ${ }^{[1,2]}$. The most common reported combination of collision tumors in ovary includes teratoma and cystadenoma or cystadenocarcinoma $^{[3]}$. In a study conducted at the Seoul National University College of Medicine, the authors reported seven pathologically proven cases of collision tumors in ovary associated with teratoma. In four of these cases there was co-existence of ovarian teratoma with cystadenoma, whereas, in the other three cases ovarian teratoma co-existed with borderline tumor, mucinous cystadenocarcinoma and dysgerminoma ${ }^{[7]}$.

Various hypotheses have been put forward regarding the occurrence of collision tumors. According to one hypothesis, the coexistence of two primary tumors in the same tissue is due to "chance of accidental meeting". The second theory suggests that the presence of the first primary tumor changes the microenvironment, thereby, giving rise to the development of second primary tumor. Yet another school of thought suggests origin of each primary tumor from a common stem cell ${ }^{[8]}$.

Most of the collision tumors of ovary are characterized by the presence of non-fatty fluid in the cyst with solid 
component ${ }^{9}$. However, in both of our cases the radiological findings suggestive of collision tumor were not obvious. Furthermore, there was no intraoperative clue pointing towards the existence of collision tumors in our patients. Both the cases were detected to have presence of collision tumors of ovary on histopathological examination.

Our cases are different in that the combination of borderline mucinous tumor with dermoid seen in one of our patients is an extremely rare entity, when compared to other collision tumors of ovary. The other collision tumor in our series had a combination of borderline papillary serous adenocarcinoma with dermoid cyst, which later developed a metachronus invasive papillary serous cystadenocarcinoma. Both the patients are alive at present and are doing well.

This two-case series highlights the need to make radiologists, surgeons and pathologists aware of this extremely rare entity, since recognition of this tumor is essential to plan proper treatment strategies based on aggressiveness or otherwise of each component of the tumor.

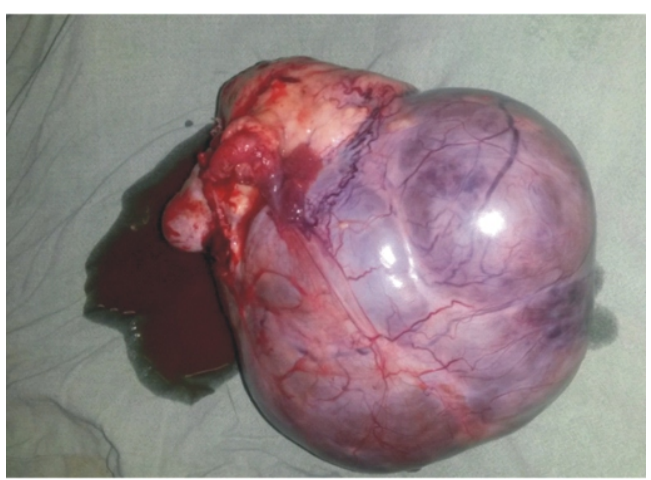

Fig.1 Surgical specimen: total abdominal hysterectomy with bilateral salpingo-oophorectomy.

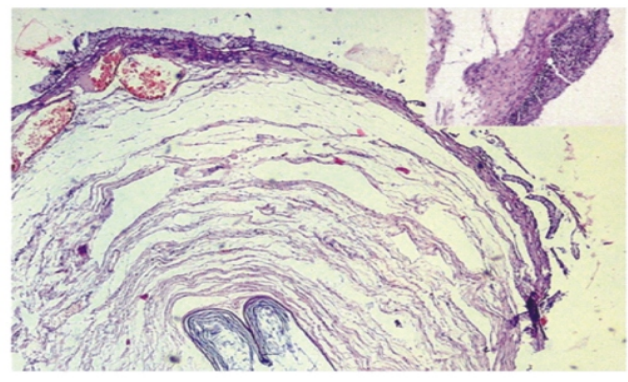

Fig.2 Photomicrograph Showing squamous epithelial lining with keratin as well as mucinousepithelial lining with mucin filled goblet cells (inset) showing stratification and overlapping. (40 x; H\&E stain).

\section{REFERENCES}

1. Bige O, Demir A, Koyuncuoglu M, Secil M, Ulukus C, Saygili U. Collision tumor: Serous cystadenocarcinoma and dermoid cyst in the same ovary. Arch Gynecol Obstet 2009; 279:767-70.

2. Singh AK, Singh M. Collision tumors of ovary: A very rare case series. J Clin Diagn Res 2014; 8: FD 14-6.

3. Papaziogas B, Souparis A, Grigoriou M et al. A rare triple co-existence of a collision tumor, a benign mature cystic teratoma and a hemorrhagic follicular cyst of the ovaries. Int J Surg 2007; 14:1-5.

4. Ozbey C, Erdogan G, Pestereli HE, Simsek T, Karaveli S. Serous papillary adenocarcinoma and adult granulosa cell tumor in the same ovary. An unusual case. APMIS 2005; 113: 713-715.

5. Dgani R, Rozenman D, Lifschitz-Mercer B. Granulosa cell tumor arising in an ovary with mature cystic teratoma. Int J Gynaecol Obstet 1993; 41: 287-289.

6. Nirenberg A, Ostör AG, Quinn MA. Collision tumor: serous adenocarcinoma and steroid cell tumor of the ovary. Pathology 1992; 24: 60-62.

7. Serung Hyup Kim, Young Jun Kin, Byung Kwan Park, Jheon Yeon cho, Bo Hyun Kin et al. Collision tumors of the ovary associated with teratomaClues to the correct pre-operative diagnosis. Journal of Computer Assisted Tomography 1999; 23;29-33.

8. Brandwein-Gensler M, Urken M, Wang B. Collision tumor of the thyroid: A case report of metastatic liposarcoma plus papillary thyroidcarcinoma. Head Neck 2004; 26: 637-41.

9. Patterson DM, Murugaesu N, Holden L, et al. A review of the close surveillance policy for stage I female germ cell tumors of the ovary and other sites. Int J Gynecol Cancer 2008; 18: 43-50. 